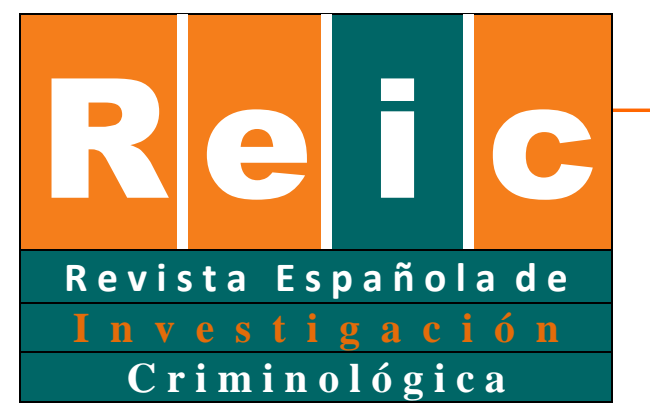

Vasilescu \& López- Riba

\title{
Diferencias por sexo entre la población penada a medidas penales alternativas en Cataluña: un análisis con perspectiva de género
}

Gender differences among the population sentenced to community sentences in Catalonia: a gender-responsive analysis

\section{Cristina Vasilescu (iD) 1}

Universidad de Girona

José María López- Riba

Universidad de Girona

\section{RESUMEN}

Los estudios realizados desde una perspectiva de género en otros países y la normativa internacional subrayan la importancia de (re)conocer las similitudes y diferencias existentes entre mujeres y hombres penados y su impacto en la ejecución de las penas. En España, a pesar de disponer de numerosas investigaciones que analizan el género en prisión, carecemos de estudios que hagan lo mismo en el sistema de medidas penales alternativas. El presente trabajo pretende conocer si existen diferencias entre las mujeres y los hombres penados a medidas penales alternativas. Para ello, se lleva a cabo un análisis bivariado que estudia la relación del sexo con variables sociodemográficas, personales, procesal-penales correspondientes a las medidas penales alternativas impuestas sobre una muestra representativa de 580 mujeres y hombres que finalizaron una medida alternativa. Los resultados señalan que existen diferencias por sexo significativas entre las diferentes variables analizadas.

Palabras clave: medidas penales alternativas, género, mujeres penadas, datos estadísticos, ejecución penal

\footnotetext{
${ }^{1}$ La correspondencia debe dirigirse a: Cristina Vasilescu en cristina.vasilescu@udg.edu
}

Revista Española de Investigación Criminológica

Artículo 3, Número 19 (2021)

https://doi.org/10.46381/reic.v19i1.443

www.criminologia.net

ISSN: 1696-9219 


\begin{abstract}
International literature shows that both the prison system and the probation system are androcentric. Studies carried out from a gender perspective in other countries and international standards highlight the importance of analyse the similarities and differences between male and female offenders and their impact on the sentences served. In our country, despite the fact that there is a great body of research analysing gender in prison, there are no studies that do the same in the probation system. This paper aims to find out whether there are gender differences among the population sentenced to community sentences and their impact on the community sentences. To this end, a bivariate analysis is carried out on a representative sample of 580 women and men who completed a community sentence. This analysis was carried out to study the relationship between gender and socio-demographic and procedural-criminal variables. The results indicate that there are significant differences by gender.
\end{abstract}

Keywords: community sentences, gender-responsive approach, women offenders, statistical data, sentence implementation

\title{
1. Introducción
}

En nuestro país, a pesar de disponer de una amplia literatura que analiza el género en el sistema penitenciario (ej. Almeda y Bodelón, 2007; Cervelló, 2006; Larrauri, 1992; 1994; Navarro, 2018; Yagüe, 2007), a excepción de Vasilescu (2020), carecemos de estudios que sitúen el género en el centro de los estudios que analizan el sistema de medidas penales alternativas (en adelante SMPA).

Los estudios que analizan el sistema penitenciario concluyen que las mujeres reclusas presentan necesidades específicas que no son atendidas en un sistema orientado mayoritariamente hacia la población masculina, dando como resultado la discriminación de la población femenina en prisión (Almeda, 2003; Cervelló, 2006; Igareda, 2006; Navarro, 2018). El uso inapropiado de la prisión para gran parte de las mujeres penadas, las circunstancias específicas de las mujeres penadas y la situación discriminatoria de las reclusas son argumentos que la literatura propone para incrementar el uso de las penas alternativas a la prisión y reducir el encarcelamiento de gran parte de las mujeres penadas (Vasilescu, 2019). No obstante, no conocemos si también existen diferencias importantes entre mujeres y hombres penados a medidas penales alternativas (en adelante MPA). Además, generalmente, los datos oficiales sobre las MPA no son desagregados por sexo.

Revista Española de Investigación Criminológica

Artículo 3, Número 19 (2021)

https://doi.org/10.46381/reic.v19i1.443

Www.criminologia.net

ISSN: 1696-9219 
Según la guía metodológica del Instituto de la Mujer (2009, p. 45), una de las formas de introducir la perspectiva de género en las estadísticas es a través de la inclusión de la variable "sexo" en el análisis. En este caso, el "sexo" ha sido la variable central a partir de la cual se han analizado las demás variables recogidas.

Así pues, el objetivo de este trabajo es establecer si existen diferencias por sexo por lo que respecta a las características sociodemográficas y procesal-penales y si estas son significativas entre la población penada a MPA como los Trabajos en Beneficio de la Comunidad (en adelante TBC) y las suspensiones de la pena de prisión con obligación de Programa Formativo (en adelante PF) en Cataluña ${ }^{2}$ en el año 2017.

El artículo se estructura en tres apartados. En primer lugar, se presenta el marco teórico y se contextualiza el estudio realizado. En segundo lugar, se presentan las diferencias por sexo de la población penada a MPA y su impacto en el cumplimiento de las medidas a través del análisis estadístico bivariado. En tercer lugar, se discuten los resultados obtenidos en relación con los objetivos y las investigaciones previas y se señalan las posibles implicaciones prácticas y las limitaciones del estudio.

\section{Contextualización del estudio y marco teórico}

En la literatura internacional, en las últimas décadas ha habido una gran proliferación de investigaciones que analizan el género -entre otras variables centrales- en la ejecución penal de MPA. La conclusión principal a la cual llegan estos estudios, es que el género marca diferencias importantes en el sistema de ejecución de MPA y que para que el tratamiento que recibe la población penada sea equitativo y no discriminatorio, es necesario reconocer dichas diferencias y específicamente, atender las necesidades de las mujeres penadas, puesto que, al igual que en prisión, las mujeres vuelven a verse discriminadas (Gelsthorpe, 2020; Malloch y McIvor, 2012; Phoenix, 2017).

\footnotetext{
${ }^{2}$ Cataluña es la única comunidad autónoma española que desde el 1 de enero de 1984 tiene transferidas las competencias en materia ejecución y supervisión de las penas, siendo estas ostentadas por el Departamento de Justicia de la Generalitat. El resto del territorio está bajo la dirección de la Secretaría General de Instituciones Penitenciarias del Ministerio del Interior (Blay, 2019).
}

Revista Española de Investigación Criminológica

Artículo 3, Número 19 (2021)

https://doi.org/10.46381/reic.v19i1.443

www.criminologia.net

ISSN: 1696-9219 
De hecho, a nivel internacional, las Reglas de Bangkok (2011) fueron pioneras en reconocer que el SMPA se había construido sobre la base de un sujeto que era masculino y que para la mayoría de las mujeres penadas eran necesarias unas MPA diseñadas en función de sus necesidades y características (Phoenix, 2018, p. 185). Como resultado de esta normativa, se han ido implementando diferentes alternativas a la prisión diseñadas para la población femenina penada alrededor del mundo (Fulham, 2019).

La literatura evidencia que las diferencias entre mujeres y hombres penados que requieren ser reconocidas y tenidas en cuenta se dan en dos niveles: en las características, necesidades y problemáticas personales que las mujeres y los hombres penados presentan, y en las características y ejecución de las MPA que tienen que cumplir.

Por lo que respecta a las características personales, se destaca que, las mujeres, en mayor medida que los hombres: a) suelen ser las cuidadoras principales de sus hijos y otras personas dependientes; b) han sido o son víctimas de cualquier tipo de violencia; c) presentan ansiedad y depresión; d) tienen a cargo un amplio conjunto de responsabilidades y cargas, sobre todo domésticas; y e) presentan falta de apoyo social y familiar (Carlen, 2012; Covington y Bloom, 2003; 2006; Malloch y McIvor, 2012; Masson y Osterman, 2017).

En cuanto a las características y ejecución de las MPA, se ha visto que las mujeres, en comparación con los hombres: a) cometen menos delitos, siendo los cometidos, relativamente leves y no violentos; b) su riesgo de reincidencia es más bajo; c) presentan un cumplimiento más irregular de las medidas; d) incumplen las MPA por diferentes razones; y e) existen diferencias entre los tipos de trabajo que realizan durante el cumplimiento de TBC, con prácticas que reproducen sus responsabilidades domésticas y perpetúan los estereotipos del rol femenino y masculino en sociedad (Dominelli, 1984; Gelsthorpe et al., 2007; Howard League for Penal Reform, 1999; Malloch y McIvor, 2012; Roberts y Watson, 2017; Wright y Kemshall, 1994; Worrall, 2003).

Ante estas características, la literatura internacional lleva más de una década apostando por la introducción de la perspectiva de género en el sistema de ejecución penal, lo que significaría partir de la necesidad, dadas las evidencias, de (re)conocer el impacto desigual que los roles de género tienen sobre las mujeres en nuestra sociedad y las diferencias

Revista Española de Investigación Criminológica

Artículo 3, Número 19 (2021)

https://doi.org/10.46381/reic.v19i1.443

www.criminologia.net

ISSN: 1696-9219 
existentes entre mujeres y hombres penados (Covington y Bloom, 2006, p. 3) para dar una respuesta más equitativa a toda la población usuaria (Wright y Kemshall, 1994).

En nuestro ámbito de estudio, mayoritariamente, las investigaciones sobre la ejecución penal de MPA, a pesar de su gran aportación a la literatura, parten de un enfoque neutral al género. De hecho, en la mayoría de ocasiones, se muestra inicialmente el porcentaje de hombres y mujeres que forman la muestra, pero no se vuelve a hacer hincapié en las posibles diferencias o similitudes. Además, generalmente, las mujeres suelen tener un papel periférico y marginal.

No obstante, existe una publicación reciente (Vasilescu, 2020) que analiza y presenta por primera vez: a) características sociodemográficas y procesal-penales desagregadas por sexo de personas penadas a MPA en Cataluña y b) buenas prácticas durante la ejecución de las MPA a partir de entrevistas a mujeres penadas y profesionales que se encargan de su supervisión. El análisis cuantitativo y cualitativo concluyen que a pesar de que existen ciertas similitudes entre mujeres y hombres penados, hay diferencias en la mayoría de las variables estudiadas que se tendrían que tener en cuenta para una ejecución equitativa. De la misma forma, las buenas prácticas señaladas por las mujeres penadas y los profesionales coinciden en gran parte con aquellas identificadas en la literatura como específicas para las mujeres.

Dado que en la literatura internacional se subraya la amplia gama de problemáticas y responsabilidades que las mujeres penadas presentan en comparación con los hombres penados y su impacto en el cumplimiento de las MPA (Worrall y Gelsthorpe, 2009; Reglas de Bangkok, 2011; Masson y Osterman 2017) y dada la falta de datos al respecto en nuestro país, el presente estudio tiene como objetivo principal llenar este vacío analizando y presentando datos primarios diferenciados por sexo sobre personas penadas a MPA.

Revista Española de Investigación Criminológica

Artículo 3, Número 19 (2021)

https://doi.org/10.46381/reic.v19i1.443

www.criminologia.net

ISSN: 1696-9219 


\section{Método}

La autorización inicial para realizar toda la investigación fue proporcionada por el Departamento de Justicia de la Generalitat de Cataluña, específicamente por el Área de Planificación y Proyectos Estratégicos de Medidas Penales Alternativas de la Dirección General de Ejecución penal a la Comunidad y de Justicia Juvenil. Se pidió acceso a los datos del Sistema Informático de Justicia Juvenil y Medidas Penales Alternativas (en adelante SIJJ/MPA). Se facilitó un usuario para explorar los datos. Dicha actividad se llevó a cabo desde los ordenadores facilitados por INTRESS, que corresponde al Área de Medidas Penales Alternativas de Girona porque dicho sistema informático tiene una base de datos propia y la única manera de recoger los datos era desde los ordenadores propios del SIJJ/MPA. Se recogió la información anonimizándola y se guardó en una base de datos de SPSS (posteriormente se utilizó R y R Studio para los análisis).

En principio, los datos que se iban a recoger correspondían a los "Descriptores estadísticos" del SIJJ/MPA, un conjunto de variables que cada expediente debía tener. No obstante, al inicio del trabajo de campo se pudo observar que dicha información no estaba presente en todos los expedientes. Entonces, se decidió igualmente recoger algunas de las variables de los descriptores, pero buscándolas en cada uno de los expedientes. Esto ha representado más de 3.000 documentos analizados, entre los cuales destacan los siguientes: el informe inicial, el informe de seguimiento, el informe final, la ficha personal, la sentencia ejecutoria y los descriptores estadísticos.

\subsection{Muestra}

Se sustrajo una muestra representativa de mujeres y hombres penados que en el año 2017 finalizaron las siguientes MPA en las provincias de Girona y Barcelona: pena de TBC, obligación de TBC, obligación de PF y obligación de tratamiento de deshabituación. La elección de estas MPA se basó en que estas implican una mayor supervisión e intervención por parte de los profesionales del SMPA (Blay y Larrauri, 2015). 
La población total de personas que en el año 2017 (desde el uno de enero de 2017 hasta el 31 de diciembre 2017) finalizó alguna de las MPA mencionadas anteriormente en dichas provincias fue de 5598 personas $(\mathrm{N}=5598)$. De esta población el 90,7\% fueron hombres y el 9,3\% mujeres.

Teniendo en cuenta que las mujeres serían un extracto de la muestra con muy poco peso y que se buscaba un análisis comparativo entre hombres y mujeres, se ha utilizado una estrategia de muestreo aleatorio estratificado con afijación igual. Se ha recogido una muestra representativa independiente de mujeres y otra de hombres, para posteriormente combinarlas. De esta forma, se ha dado el mismo nivel de importancia a los dos grupos. Esto ha permitido analizar y comparar las características de cada colectivo con una mayor precisión. Para calcular la muestra se marcó un nivel de confianza del 95\% y un margen de error del 5\%. Así se obtuvo una muestra representativa de la población femenina (519) de $n=222$ y de la población masculina (5179) de $n=358$. Por lo tanto, la muestra final es de 580 personas $(n=580)$.

Para seleccionar los sujetos de cada estrato se ha realizado una selección sistemática (Ruiz, 2012). Esto se refiere a que se ha seleccionado al azar el primer individuo y los restantes a intervalos fijos hasta que se ha llegado al total del número de la muestra que se ha querido conseguir. Para conocer los intervalos fijos, por una parte, se ha dividido el universo de mujeres por la muestra de estas (519/222) y, por otra parte, el universo de hombres por la muestra de estos (5079/358).

\subsection{Variables}

En este apartado se presentan las variables que se utilizaron en los análisis del presente trabajo. Por una parte, estas variables hacen referencia a las características sociodemográficas y procesal-penales de las personas penadas, así como al tipo de MPA impuesta y características de la ejecución de los TBC y PF. La elección de estas variables personales y penales se basa, principalmente, en la importancia que se les ha dado en la literatura previa.

Revista Española de Investigación Criminológica

Artículo 3, Número 19 (2021)

https://doi.org/10.46381/reic.v19i1.443

www.criminologia.net

ISSN: 1696-9219 


\section{Variables sociodemográficas}

Sexo. Se recogió una variable que indicaba si el sexo de la persona era mujer $(38,62 \%)$ u hombre (61,38\%). Las opciones limitadas de las categorías mujer/hombre de la variable sexo se dan por el binarismo de la base de datos del SMPA, puesto que la información que se podía recoger a través de la base de datos era solamente si la persona era hombre o mujer.

País de origen. Esta variable tiene dos categorías: España $(68,62 \%)$ y Extranjero $(31,32 \%)$. La elección de la variable país de origen en vez de nacionalidad implica que de las personas que se han recogido como originarias de un país extranjero, hay personas que tienen la nacionalidad española en el momento de la recogida de datos. Se ha considerado interesante recoger información sobre el lugar de procedencia de la persona, por considerarse por sus rasgos sociológicos personas más vulnerables y con mayor probabilidad de ser criminalizadas por el sistema penal a pesar de tener la nacionalidad española (Philips et al., 2019).

\section{Situación personal}

Situación sentimental. Se recogió información para crear una variable que indicara si la persona estaba soltera $(49,83 \%)$ o en pareja $(45,34 \%)$. No se obtuvo información sobre esta variable para el 4,83\% de la muestra. Cabe señalar que dicha variable no mide la convivencia o no convivencia de la persona penada con su pareja.

Hijos/as o familiares a cargo. Se quiso contar con una variable que recogiera información sobre si la persona penada tenía hijos/as o familiares a cargo. En un 63,1\% de los casos no se contaba con hijo/as o familiares a cargo, mientras que en un 26,21\% sí. En un $10,69 \%$ de los casos no se pudo establecer.

Apoyo social/familiar. A través de los diferentes informes sociales y la ficha personal se construyó una variable para conocer si la persona recibía apoyo por parte de algún familiar, pareja y/o amistades (66,03\%). En aquellos casos en los que se entendía que la persona carecía de apoyo alguno se marcaba la opción "no" (30,17\%). En un 3,79\% de los casos no se pudo determinar la existencia o ausencia de apoyo social o familiar.

Revista Española de Investigación Criminológica

Artículo 3, Número 19 (2021)

https://doi.org/10.46381/reic.v19i1.443

Www.criminologia.net

ISSN: 1696-9219 
Víctima de algún tipo de violencia. Esta variable se entendió en un sentido amplio, puesto que se recogían en la opción del "sí" $(18,45 \%)$ a todas aquellas personas penadas que en el presente o en el pasado habían sido víctimas de cualquier tipo de violencia, ya fuera esta psíquica y/o física. Un 77,76\% de las personas penadas no habían sido víctima de ningún tipo de violencia. Mientras que para un 3,79\% no se pudo determinar si habían sido víctimas.

Enfermedad mental y/o física. En esta variable se incluye a todas aquellas personas que en el momento del cumplimiento de la medida presentaban algún tipo de enfermedad mental y/o física y así se identificara en los informes. Lo que ocurrió en un 39,31\% de los casos. Mientras que un 58,45\% de las personas penadas no presentaban ningún tipo de enfermedad. Para un 2,24\% no se pudo establecer la existencia o ausencia de algún tipo de enfermedad.

\section{Variables procesal-penales}

Antecedentes penales. Esta variable no se recoge directamente en la base de datos del SMPA. Se pudo averiguar leyendo la sentencia donde explícitamente se menciona el hecho de si la persona tiene $(41,21 \%)$ o carece $(58,79 \%)$ de antecedentes penales (computables o no a efectos de reincidencia). La información se comprobó en segunda instancia tras la lectura del expediente de la persona en el SMPA para ver si había estado vinculada para cumplir otra medida o bien tenía que cumplir otra medida por otra sentencia ejecutoria y/o buscando la persona en la base de datos del Servicio Penitenciario de la Generalitat de Cataluña (aunque en este caso solamente se refiere a si la persona ha estado o está en alguna prisión catalana).

Delito. Para resumir la información en el mínimo de categorías posible de cara a hacer los análisis más fácilmente interpretables se construyeron tres categorías: Seguridad vial (48,1\%), violencia de género o doméstica (en adelante VIGE/VIDO) (21,21\%) y otros $(30,69 \%)$. Dentro de esta última categoría se recogen delitos contra el patrimonio, delitos contra salud pública, delitos contra las personas, delitos contra la autoridad, delitos contra la libertad sexual y daños, entre otros.

Revista Española de Investigación Criminológica

Artículo 3, Número 19 (2021)

https://doi.org/10.46381/reic.v19i1.443

Www.criminologia.net

ISSN: 1696-9219 


\section{Características de las MPA impuestas}

Tipo de MPA. Aquí se recogen tres categorías: TBC (82,24\%), PF (13,1\%) y Tratamiento de deshabituación $(4,66 \%)$.

Tipo de TBC. En este caso, se recogió información sobre el tipo concreto de TBC que la persona penada tenía que realizar y se resumió en las siguientes categorías: Trabajo: mantenimiento/limpieza (49,06\%), Trabajo: Tareas administrativas (18,45\%), Trabajo: tareas de cuidados (14,05\%), Trabajo: otros (6,29\%), Actividades formativas $(1,05 \%)$, Tratamiento $(5,87 \%)$. Para un 5,24\% de la muestra no se pudo identificar el Tipo de TBC concreto. Cabe decir que el TBC se puede realizar en formato formativo, de trabajo, de tratamiento o mixto. Antes de la aprobación del Real Decreto 840/2011, el contenido de los TBC era estricto porque solamente cabía la posibilidad de realizar trabajos de utilidad social y pública o de participar en un taller de seguridad vial. Con el nuevo decreto este contenido se amplía, y la persona penada que deba cumplir un TBC lo puede hacer no solamente mediante trabajo sino, además, mediante formación (participando en talleres o programas formativos de carácter cultural, laboral, educación sexual, educación vial, entre tantos otros) y terapia (tratamiento drogas, salud mental y/o trastorno alimentario) o mixto.

Asistencia TBC. Se construyó una variable que indicaba si la persona penada a un TBC asistía de forma satisfactoria (Asistencia: 63,31\%), de forma irregular (Asistencia irregular: $15,72 \%$ ) o no asistía a sus tareas (No asistencia: 20,96\%). La categoría "Asistencia irregular" recoge las faltas de puntualidad, las faltas de asistencia y la muestra de una actitud negativa durante el cumplimiento. La categoría de "No asistencia" se corresponde a aquellas personas que han abandonado la medida y se encuentran en busca y captura, las que han cometido un nuevo delito y por tanto se les ha revocado la pena, y las que han entrado en prisión por otro delito.

Motivo no asistencia TBC. Para aquellas personas que se encontraban en la categoría de "No asistencia" de la variable anterior se recogió además el motivo de esta. Estos motivos se agruparon en dos categorías: Incumplimiento (45\%) y Otros motivos (54\%). Dentro de la categoría "Otros motivos" se recoge: prescripción de la pena, pago de la multa, imposibilidad por problemas graves de salud, derivación a otras comunidades autónomas y defunción de la

Revista Española de Investigación Criminológica

Artículo 3, Número 19 (2021)

https://doi.org/10.46381/reic.v19i1.443

www.criminologia.net

ISSN: 1696-9219 
persona penada. En el caso de una persona (1\%) no se pudo determinar el motivo de la no asistencia.

Tipo PF. Esta variable recoge tres categorías: Seguridad vial $(36,84 \%)$, VIGE/VIDO $(48,68 \%)$ y Otros $(14,47 \%)$. Dentro de esta última categoría se incluyen los programas formativos de educación sexual, educación derechos humanos, conductas violentas, inserción laboral, medio ambiente, alfabetización e idiomas.

Asistencia PF. De la misma manera que se hizo con el TBC, se registró información sobre la asistencia a PF. En este caso, un $67,11 \%$ de los penados asistieron con normalidad al PF (Asistencia), un 19,74\% asistió de forma irregular (Asistencia irregular) y un 13,16\% no asistió (No asistencia).

\subsection{Estrategia de análisis}

En primer lugar, en base a los datos presentados en el apartado anterior se puede realizar una primera aproximación al perfil de persona penada a MPA en Girona y Barcelona. Con mayor probabilidad, la persona cumpliendo una MPA sería una persona con pareja, sin hijos/as y/o familiares a cargo, que cuenta con apoyo familiar/social, que no ha sido víctima de violencia ni presenta alguna enfermedad física y/o mental, sin antecedentes penales, que ha cometido un delito contra la seguridad vial.

Sin embargo, el objetivo del presente trabajo era conocer las diferencias de sexo. Por ello, se han llevado a cabo diferentes análisis bivariados para conocer la relación del sexo con el resto de las variables. Esto se ha hecho mostrando la distribución relativa de cada sexo en cada una de las categorías de las otras variables. Para ver si estas diferencias son estadísticamente significativas se han realizado pruebas de Chi cuadrado.

\section{Resultados}

\subsection{Análisis bivariados}

Para dar respuesta al objetivo del trabajo se han realizado diferentes análisis bivariados. Esto permite conocer la relación del sexo con el resto de las variables. La siguiente tabla muestra la distribución relativa de cada sexo en cada una de las categorías de las otras variables y el

Revista Española de Investigación Criminológica

Artículo 3, Número 19 (2021)

https://doi.org/10.46381/reic.v19i1.443

www.criminologia.net

ISSN: 1696-9219 
resultado de las pruebas de Chi cuadrado que se han llevado a cabo para ver si estas diferencias son estadísticamente significativas.

\section{Tabla 1.}

Análisis bivariado

\begin{tabular}{|c|c|c|c|c|c|}
\hline Variable & Categoría & $\begin{array}{c}\text { Hombre } \\
(\%)\end{array}$ & $\begin{array}{c}\text { Mujer } \\
(\%)\end{array}$ & $\begin{array}{c}\text { Chi } \\
\text { cuadrado }\end{array}$ & $\mathbf{p}$ \\
\hline \multirow{2}{*}{ País de origen } & Español & 68,8 & 68,3 & \multirow{2}{*}{0,002} & \multirow{2}{*}{0,97} \\
\hline & Extranjero & 31,2 & 31,7 & & \\
\hline \multirow{2}{*}{ Situación sentimental } & Soltero/a & 48,1 & 59,2 & \multirow{2}{*}{5,99} & \multirow{2}{*}{0,01} \\
\hline & En pareja & 51,9 & 40,8 & & \\
\hline \multirow{2}{*}{$\begin{array}{l}\text { Hijos/as o familiares } \\
\text { a cargo }\end{array}$} & No & 86,6 & 44,7 & \multirow{2}{*}{101,53} & \multirow{2}{*}{$<0,000$} \\
\hline & Sí & 13,4 & 55,3 & & \\
\hline \multirow{2}{*}{ Apoyo familiar/social } & No & 17,2 & 54 & \multirow{2}{*}{81,23} & \multirow{2}{*}{$<0,000$} \\
\hline & Sí & 82,8 & 46 & & \\
\hline \multirow{2}{*}{ Víctima de violencia } & No & 96,8 & 54,5 & \multirow{2}{*}{148,96} & \multirow{2}{*}{$<0,000$} \\
\hline & Sí & 3,2 & 45,5 & & \\
\hline \multirow{2}{*}{$\begin{array}{l}\text { Enfermedad } \\
\text { mental/física }\end{array}$} & No & 70,8 & 42,2 & \multirow{2}{*}{44,38} & \multirow{2}{*}{$<0,000$} \\
\hline & Sí & 29,2 & 57,8 & & \\
\hline \multirow{2}{*}{ Antecedentes penales } & No & 51,4 & 70,5 & \multirow{2}{*}{19,99} & \multirow{2}{*}{$<0,000$} \\
\hline & Sí & 48,6 & 29,5 & & \\
\hline \multirow{3}{*}{ Delito cometido } & Seguridad vial & 43,8 & 54,9 & \multirow{3}{*}{74,89} & \multirow{3}{*}{$<0,000$} \\
\hline & VIGE/VIDO & 32,6 & 3,1 & & \\
\hline & Otro & 23,6 & 42 & & \\
\hline \multirow{3}{*}{ Tipo MPA } & ТВC & 77 & 90,6 & \multirow{3}{*}{20,15} & \multirow{3}{*}{$<0,000$} \\
\hline & $\mathrm{PF}$ & 18 & 5,4 & & \\
\hline & Tratamiento deshabituación & 5 & 4 & & \\
\hline \multirow{6}{*}{ Tipo de TBC } & Trabajo: mantenimiento/limpieza & 68,7 & 28,4 & \multirow{6}{*}{89,13} & \multirow{6}{*}{$<0,000$} \\
\hline & Trabajo: tareas administrativas & 7,3 & 36,4 & & \\
\hline & Trabajo: tareas de cuidados & 11 & 20 & & \\
\hline & Trabajo: otros & 6,5 & 6,8 & & \\
\hline & Actividades formativas & 0,8 & 1,6 & & \\
\hline & Tratamiento & 5,7 & 6,8 & & \\
\hline
\end{tabular}




\begin{tabular}{|c|c|c|c|c|c|}
\hline \multirow{3}{*}{ Asistencia TBC } & Asistencia & 67,5 & 57,6 & \multirow{3}{*}{5,85} & \multirow{3}{*}{0,05} \\
\hline & Asistencia irregular & 12,8 & 19,7 & & \\
\hline & No asistencia & 19,7 & 22,7 & & \\
\hline \multirow{2}{*}{$\begin{array}{l}\text { Motivo no asistencia } \\
\text { TBC }\end{array}$} & Incumplimiento & 51,9 & 38,3 & \multirow{2}{*}{1,34} & \multirow{2}{*}{0,25} \\
\hline & Otros motivos & 48,1 & 61,7 & & \\
\hline \multirow{3}{*}{ Tipo PF } & Seguridad vial & 29,7 & 75 & \multirow{3}{*}{9,01} & \multirow{3}{*}{0,01} \\
\hline & VIGE/VIDO & 54,7 & 16,7 & & \\
\hline & Otros & 15,6 & 8,3 & & \\
\hline \multirow{3}{*}{ Asistencia PF } & Asistencia & 73,4 & 33,3 & \multirow{3}{*}{13,43} & \multirow{3}{*}{0,001} \\
\hline & Asistencia irregular & 12,5 & 58,3 & & \\
\hline & No asistencia & 14,1 & 8,3 & & \\
\hline
\end{tabular}

En primer lugar y por lo que respecta al país de origen las diferencias no son significativas. Por lo que respecta a la situación sentimental, se pueden ver claras diferencias por sexo, dichas diferencias son estadísticamente relevantes. Un 59,2\% de las mujeres de la muestra estaban solteras en el momento del cumplimiento de la MPA, en comparación con el 48,1\% de los hombres en la misma situación.

La variable hijos/as a cargo y/u otros familiares también muestra diferencias por sexo significativas. Un 55,3\% de las mujeres de la muestra tienen hijas/os a cargos y/u otros familiares en comparación con el 13,4\% de los hombres en la misma situación. Además, si tenemos en cuenta que más de la mitad de las mujeres estaban solteras, cabe deducir que muchas de ellas se encontraban solas con la responsabilidad del cuidado de los hijos durante el cumplimiento de la MPA. En concreto un 28,57\% de mujeres se encontraban en esta situación frente a un 2,53\% de los hombres.

Por lo que respecta el apoyo social/familiar, destaca que más de la mitad de las mujeres de la muestra (54\%) carece de este tipo de apoyo en comparación con el 17,2\% de los hombres de la muestra en la misma situación, siendo esta diferencia significativa.

Esta tabla también nos muestra otro dato preocupante y es que casi la mitad de las mujeres de la muestra $(45,5 \%)$ ha sido víctima de violencia física, psíquica y/o sexual durante su trayectoria vital o en el momento del cumplimiento de la medida, en comparación con el 
$3,2 \%$ de los hombres que han sufrido algún tipo de violencia. Durante el trabajo de campo se pudo observar que, mayoritariamente, dicha violencia fue llevada a cabo por parte de parejas o ex parejas del género masculino o por algún familiar, en su gran mayoría, también hombres.

En la variable enfermedades físicas y/o mentales, vuelven a resaltar las diferencias por sexo que, además, son significativas. En este sentido, destaca el importante porcentaje de mujeres $(57,8 \%)$ que presentaba algún tipo de enfermedad mental (sobre todo ansiedad, depresión y estrés post traumático) y/o física durante el cumplimiento de la MPA en comparación con los hombres de la muestra $(29,2 \%)$. Aunque el porcentaje de hombres con enfermedad es alto, la diferencia es casi de 30 puntos respecto a las mujeres. Además, cabe señalar que en muchas ocasiones las personas presentaban tanto una enfermedad mental (o múltiples) como una enfermedad física.

Las distintas variables procesal-penales también presentan diferencias por sexo. Por lo que respecta el historial delictivo, los hombres penados a MPA presentan antecedentes penales en mayor medida que las mujeres ( $48,6 \%$ y $29,5 \%$ respectivamente) y, además, dicha diferencia es estadísticamente significativa. El tipo de delito cometido también muestra diferencias por sexo significativas. Gran parte de las mujeres penadas a MPA cumplen condena por delitos contra la seguridad vial $(54,9 \%)$, mientras que los hombres, a pesar de cumplir gran parte de sus condenas por delitos de la misma índole (43,8\%), también cumplen MPA por delitos de violencia de género y violencia doméstica en mayor medida que las mujeres (32,6\% frente a $3,1 \%)$.

Por lo que respecta el tipo de MPA impuesta, destaca que al 90,6\% de las mujeres se le impuso TBC, tratándose de un porcentaje ligeramente superior que en el caso de los hombres (77\%). En cuanto al PF, la segunda medida más impuesta, sobresale el porcentaje de hombres (18\%) al cual se le impuso PF que triplica el porcentaje de las mujeres $(5,4 \%)$ al cual se le impuso la misma medida. Además, dichas diferencias también son estadísticamente significativas.

Como se puede observar, existen diferencias significativas en el tipo de TBC que cumplen mujeres y hombres. En este sentido, la tabla muestra que existen diferencias significativas en el tipo de TBC, puesto que las mujeres realizan más tareas administrativas

Revista Española de Investigación Criminológica

Artículo 3, Número 19 (2021)

https://doi.org/10.46381/reic.v19i1.443

www.criminologia.net

ISSN: 1696-9219 
$(36,4 \%)$ y de cuidado (20\%) y los hombres de mantenimiento (68,7\%). En este sentido, es interesante destacar que, a pesar de que la categoría "mantenimiento y/o limpieza" recoge dichas tareas conjuntamente en la base de datos del SIJJ/MPA, en los informes y fichas de las entidades de TBC se pudo observar que en el caso de los usuarios predominaban las actividades de mantenimiento frente a las de limpieza y en el caso de las usuarias, aquellas de limpieza frente las de mantenimiento.

La asistencia al TBC también presenta diferencias por sexo que además son estadísticamente significativas. Destaca que, a pesar de que más de la mitad de la población de la muestra asistió al TBC, siendo el porcentaje de los hombres (67,5\%) ligeramente superior al de las mujeres $(57,6 \%)$, hay una gran parte de la población que no asistió o que presentó una asistencia irregular. En primer lugar, las mujeres presentan una mayor asistencia irregular al TBC (19,7\%) en comparación con los hombres (12,8\%). En segundo lugar, sobresale el alto porcentaje de población tanto femenina $(22,7 \%)$ como masculina $(19,7 \%)$ que no asistió finalmente al TBC impuesto.

En cuanto al tipo de PF, destaca que la mayoría de mujeres realizan programas formativos relacionados con la seguridad vial (75\%) mientras que los hombres se reparten entre los de seguridad vial $(29,7 \%)$ y los relacionados con la VIGE/VIDO (54,7\%). Estas diferencias son significativas, y seguramente reflejen las diferencias en los delitos cometidos señaladas anteriormente. En la asistencia a estos programas también se observan diferencias estadísticamente significativas en cuanto al sexo, puesto que, las mujeres, como en el caso del TBC, tienen una asistencia irregular mayor que los hombres (58,3\% frente a $12,5 \%)$, sin embargo, estos no asisten a los programas en mayor medida que las mujeres $(14,1 \%$ frente a $8,3 \%)$.

En suma, parece ser que las mujeres penadas presentan más problemáticas y necesidades en comparación con los hombres penados a MPA y que estas pueden influir en la asistencia al TBC y al PF. 


\section{Discusión y conclusiones}

El objetivo de esta investigación era conocer si existían diferencias por sexo por lo que respecta a las características sociodemográficas, procesal-penales y referentes al cumplimiento de las MPA y si estas eran significativas entre la población penada a medidas alternativas como los TBC y las suspensiones de la pena de prisión con obligación de PF en el año 2017 en Cataluña.

Una de las características de realizar investigación criminológica con perspectiva de género, es problematizar el género, es decir, tomar conciencia de su importancia a lo largo del análisis y discutir las posibles implicaciones que podría tener en la vida de las personas penadas y en este caso, en la ejecución de las MPA (Daly y Chesney-Lind, 1988; Smart, 1976). En la misma línea, una de las teorías pioneras que justifican la necesidad de la introducción de la perspectiva de género dentro del sistema de ejecución penal es la de "gendered pathways" de Covington y Bloom (2006), que destaca que existen ciertas características y experiencias previas específicas al género femenino que, en muchas ocasiones, implican un impacto diferente y desproporcional del tratamiento recibido durante el cumplimiento de las medidas.

En este sentido, cabe subrayar que el análisis bivariado realizado es un reflejo del impacto que tiene la socialización de género en la vida de las mujeres penadas. Los resultados del estudio han mostrado que sí existen diferencias por sexo significativas entre la población penada a MPA.

En lo que se refiere a las características sociodemográficas y personales, las mujeres penadas, en mucha mayor medida que los hombres penados, están solteras, se encuentran al cargo de sus hijos/as y/u otros familiares sin tener apoyo familiar y/o social, han sido víctimas de violencia y presentan algún tipo de enfermedad física y/o mental.

Gran parte de las mujeres de la muestra se encargan del cuidado de sus hijos u otros familiares, en muchas ocasiones, siendo madres solteras, una imagen muy similar a la de la gran mayoría de mujeres presas (Navarro, 2018). Este dato se suma a los hallazgos de otros países donde la gran mayoría de las mujeres que deben cumplir una medida son las encargadas de cuidar de los hijos y/o de otras personas dependientes, lo que dificulta en

Revista Española de Investigación Criminológica

Artículo 3, Número 19 (2021)

https://doi.org/10.46381/reic.v19i1.443

Www.criminologia.net

ISSN: 1696-9219 
muchas ocasiones el cumplimiento regular con las medidas impuestas (Sheehan et al., 2007; 2011; Gelsthorpe et al., 2007; Barr, 2019; Fulham 2019; Gelsthorpe, 2020).

Dichos hallazgos también coinciden con la evidencia que subraya que, generalmente, muchas de las mujeres penadas presentan una gran falta de apoyo social/familiar, sobre todo desde su entrada en el sistema de justicia penal (Gelsthorpe et al., 2007; Prison Reform Trust, 2018; Barr, 2019). Además, las evidencias concluyen que el apoyo social/familiar es uno de los elementos más decisivos en el cumplimiento exitoso de una MPA y en el abandono del delito de las mujeres penadas (Covington y Bloom, 2003; McIvor, Trotter y Sheehan, 2009; Penal Reform International, 2016).

Un dato preocupante es, de forma similar a otros países, el número de penadas que han sido víctimas de violencia por parte de sujetos masculinos a lo largo de su vida o en el momento del cumplimiento de la MPA (Covington y Bloom, 2003; Gelsthorpe et al., 2007; Burgess, Malloch y McIvor, 2011; Reglas de Bangkok, 2011; Fulham, 2019). Esta es una característica específica del género femenino (Cruells e Igareda, 2005; Covington y Bloom, 2003; 2006), que, en palabras de Navarro (2018, p. 124) "se repite más allá de nuestras fronteras".

Otro dato que llama la atención es la proporción de mujeres penadas que presentan enfermedades físicas y/o mentales en el momento del cumplimiento de las MPA. En la misma línea que otros países, destaca la alta población de mujeres con necesidades de bienestar emocional en el sistema penal (Masson y Osterman, 2017; Annison et al., 2018; Barr, 2019; Petrillo, 2019). Según la teoría del trauma (Covington y Bloom, 2006) -otra de las teorías que justifican la introducción de la perspectiva de género en el sistema de ejecución penal-, en muchas ocasiones, el trauma y la mayor probabilidad de las mujeres penadas de sufrir ansiedad y depresión se relaciona con los abusos y la violencia sufrida a lo largo de su vida. Por ello, es importante entender el proceso del trauma antes de intervenir con las mujeres penadas.

Por otra parte, destaca que gran parte de las personas de la muestra ejecutaron el TBC mayoritariamente a través de una actividad de trabajo, lo que resulta cuestionable en el caso de la población usuaria femenina dadas las múltiples problemáticas y necesidades que

Revista Española de Investigación Criminológica

Artículo 3, Número 19 (2021)

https://doi.org/10.46381/reic.v19i1.443

www.criminologia.net

ISSN: 1696-9219 
presenta (Vasilescu, 2020). También en el caso de la población usuaria masculina porque hay una gran parte que está cumpliendo TBC por delitos de violencia de género o doméstica, pudiendo optar por otras alternativas más adecuadas teniendo en cuenta el tipo de delito cometido $^{3}$. En las diferentes actividades de trabajo que realiza la población usuaria también hay que señalar las diferencias por sexo que muestran una imagen muy similar a otros contextos donde destacan las diferencias estereotipadas en las diferentes tareas (Dominelli, 1984; Worrall, 2003; McIvor, 1998; 2007; Barr, 2019). Estas se ven reflejadas en la asignación ${ }^{4}$ de las mujeres penadas a entidades que implican la prestación de servicios administrativos, de limpieza y/u orientado hacia las personas y la asignación de los hombres penados a entidades que implican mayoritariamente realizar tareas de mantenimiento. En todo caso, las razones existentes tras este tipo de vinculación y derivación pueden ser múltiples y se están analizando a partir de datos cualitativos en otro estudio. No obstante, sean cuales sean, sería necesario romper con estos estereotipos (Acale, 2019) y en ambos casos, abrir el amplio abanico de actividades de trabajo que cada persona podría realizar mientras cumple un TBC.

Por tanto, parece ser que sí existen diferencias por sexo por lo que respecta a las características sociodemográficas, personales y de cumplimiento de TBC y que, además estas son en su mayoría significativas. Además, es interesante destacar que, cuando se analiza el perfil de la mujer penada a MPA, este se muestra bastante diferente al perfil general de la persona penada a MPA que se presentaba anteriormente. Se trataría de una mujer española, soltera, con hijos/as a cargo y/u otros familiares, sin apoyo familiar/social, que ha sido víctima de violencia y que presenta algún tipo de enfermedad física y/o mental. Esta diferencia es un reflejo de la importancia que tiene recoger, analizar y presentar los datos desagregados por sexo.

Adicionalmente, parece ser que, al igual que en otros países, el cumplimiento del TBC y el PF es más problemático para las mujeres, puesto que presentan una asistencia irregular

\footnotetext{
3 Para una discusión en profundidad sobre esta cuestión véase Medina (2005) y Blay (2007).

${ }^{4}$ Generalmente, es el Delegado de Medidas Penales Alternativas el que asigna la entidad a la persona penada, aunque es cierto que, cuando es posible, esto se hace en función de su formación y experiencias laborales previas por lo que podría ser que dicha elección tenga que ver con las tareas que conoce la persona penada.
} 
mayor que los hombres. Si se tienen en cuenta las múltiples limitaciones y necesidades que las mujeres presentan en comparación con los hombres, es lógico pensar que el cumplimiento sea más irregular en el caso de las usuarias. Profundizando, en los motivos de la no asistencia en el caso de los TBC parece ser que los hombres de la muestra que no asisten al TBC lo hacen por incumplimiento de la medida mientras que las mujeres por otros motivos diferentes, aunque dicha diferencia no es estadísticamente significativa. En este sentido, la falta de un diseño o de una implementación del TBC con perspectiva de género podría ser una de las razones principales (Patel y Stanley, 2008; Easton y Matthews, 2011; Gelsthorpe, 2013; Sapouna, Bisset, Conlong y Matthews, 2015).

De todos modos, cabe señalarse que el estudio cuenta con limitaciones que provienen principalmente de las características de la muestra, por su representatividad en algunas MPAs y por los datos perdidos a causa de la limitada información que se recoge en la base de datos del SIJJ/MPA. En este sentido, el presente estudio muestra la relevancia que tiene recoger de manera uniforme información sobre variables como las estudiadas, puesto que esta información ha demostrado ser importante y necesaria en aquellos sistemas de ejecución de medidas sensibles al género (Gelsthorpe et al., 2007).

De la misma manera, el valor de los datos aquí recogidos y analizados es innegable. Los resultados obtenidos en esta investigación aportan por primera vez en España información relevante por lo que respecta la población penada a MPA y las implicaciones derivadas de las diferencias por sexo que hombres y mujeres presentan. Además, los resultados del estudio reflejan la importancia de recoger, analizar y presentar datos desagregados por sexo. A pesar de que la población usuaria del SMPA es mayoritariamente masculina, eso no debe seguir justificando la invisibilidad de las mujeres penadas. El análisis de los datos estadísticos recogidos muestra que desafortunadamente, las características, problemáticas y necesidades de la población femenina se repiten más allá de las fronteras angloamericanas. No obstante, para conocer en profundidad las implicaciones de estas características y problemáticas, es necesario complementar dicho análisis con técnicas cualitativas para conocer las experiencias de las mujeres durante el cumplimiento de las MPA y la de aquellos profesionales que se encargan de su supervisión e intervención.

\section{Revista Española de Investigación Criminológica}


Por tanto, parece ser que en la misma línea que en las investigaciones realizadas en el ámbito internacional, las mujeres de la muestra presentan una gama compleja de problemáticas y responsabilidades en comparación con sus homólogos masculinos que podrían influir en el cumplimiento de las medidas (Galbraith, 2004; Worrall y Gelsthorpe, 2009; Reglas de Bangkok, 2011; Joiner, 2011; Nugent y Loucks, 2011; Burgess et al., 2011; Carlen, 2012; Masson y Osterman, 2017; Phoenix, 2018).

El sistema de justicia penal necesita tener en cuenta las implicaciones prácticas de estos resultados. La complejidad en la ejecución penal debería tenerse en cuenta y al igual que en otros países (Sheehan, McIvor y Trotter, 2007; 2011; Phoenix, 2017; Vasilescu, 2019; Gelsthorpe et al., 2007; Gelsthorpe, 2020) donde se han demostrado como buenas prácticas específicas con las mujeres penadas ${ }^{5}$, apostar, entre otros, por: i) el TBC terapéutico o mixto para aquellas personas que presenten problemáticas de salud mental y/o físicas y para aquellas personas que hayan sido o estén siendo víctimas de violencia de algún tipo; ii) ofrecer apoyo práctico para el cuidado de los hijos/as a cargo; iii) evitar la realización de TBC que perpetúen la feminización de la pobreza y los roles de género; iv) apostar por un estilo de supervisión más flexible y comprensible que tenga en cuenta la posible dificultad de compaginar las responsabilidades y cargos con el cumplimiento de la MPA y las otras necesidades y problemáticas presentes; v) la vinculación a servicios que ofrezcan apoyo práctico; vi) contemplar la posibilidad de que las mujeres penadas no se sientan cómodas cumpliendo cualquier tipo de MPA cuando la mayoría de la población usuaria ser masculina.

En suma, este trabajo ha aportado conocimiento desagregado por sexo sobre la población penada a MPA, que ayudará a (re)conocer las posibles similitudes y diferencias, facilitará un cumplimiento cada vez más equitativo y evitará que este suponga una complicación extra para las mujeres penadas a MPA (Gelsthorpe et al., 2007; Gelsthorpe, 2020). En la misma línea, es necesario continuar investigando y conocer las experiencias de las mujeres y el impacto que tiene en sus vidas el cumplimiento de las MPA.

\footnotetext{
${ }^{5}$ Véase Vasilescu (2019) para una revisión de las propuestas que se han llevado a cabo internacionalmente y la discusión acerca de su adaptación al sistema penal español. 


\section{Referencias}

Acale, M. (2019). Penal and Custodial Control of Female Criminality in Spain from a Gender Perspective. Social Sciences, 52(8), 1-15. https://doi.org/10.3390/socsci8020052

Almeda, E. (2003). Corregir y castigar: el ayer y hoy de las cárceles de mujeres. Edicions Bellaterra.

Almeda, E., y Bodelón, E. (2007). Mujer y castigo: un enfoque socio-jurídico y de género. Dykinson.

Barry, M., y McIvor, G. (2010). Professional decision making and women offenders: containing the chaos?. Probation Journal, 57(1), 27-41. https://doi.org/10.1177/0264550509346193

Barr, Ú. (2019). Desisting Sisters: Gender, Power and Desistance in the Criminal (In)Justice System. Critical Criminological Perspectives, Palgrave Macmillan.

Blay, E. (2007). El Trabajo en Beneficio de la Comunidad como pena para la violencia familiar, Revista de Derecho Penal y Criminología, (19), 397-426.

Blay, E. (2019). El papel de los Delegados de Ejecución en la ejecución penal en la comunidad ¿gestores o agentes de rehabilitación?. InDret, Revista para el Análisis del Derecho, (4), 1-32.

Blay, E., y Larrauri, E. (2015). Community punishments in Spain: a tale of two adminitrations. En G. Robinson y F. McNeill (Eds.), Community Punishments: European perspectives (pp. 191-202). Routledge.

Burgess, C., Malloch, M., y McIvor, G. (2011). Women in focus: an evaluation. A report for South West Scotland Community Justice Authority. The Scottish Centre for Crime \& Justice Research. https://www.sccjr.ac.uk/publications/women-in-focus-anevaluation/

Carlen, P. (2012). Women's imprisonment: an introduction to the Bangkok Rules. Revista Crítica Penal y Poder, (3), 148-157.

Cervelló, V. (2006). Las prisiones de mujeres desde una perspectiva de género. Revista General de Derecho Penal, (5), 1-24.

Clarke, R. (2004). “What works?” for Women Who Offend: A Service User's Perspective: Exploring the Synthesis Between What Women Want and What Women Get. The Griffins Society. Social Policy Department, London School of Economics and Political

Science. https://www.thegriffinssociety.org/system/files/papers/fullreport/research_paper_20 04_04_r.clarke.pdf

Covington, S., y Bloom, B. (2003). Gendered Justice: Women in the Criminal Justice System. En B. Bloom, (Ed.), Gendered Justice: Addressing Female Offenders (pp. 1-20), Carolina Academic Press.

Covington, S., y Bloom, B. (2006). Gender-Responsive Treatment and Services in Correctional Settings. Women \& Therapy, 29(3-4), 9-33. https://doi.org/10.1300/J015v29n03 02

Daly, K., y Chesney-Lind, M. (1988). Feminism and Criminology. Justice Quarterly Academy of Criminal Justice Sciences, 5(4), 497-538. https://doi.org/10.1080/07418828800089871

Revista Española de Investigación Criminológica

Artículo 3, Número 19 (2021)

https://doi.org/10.46381/reic.v19i1.443

www.criminologia.net

ISSN: 1696-9219 
Dominelli, L. (1984). Differential Justice: Domestic Labour, Community Service and Female Offenders. Probation Journal, 31(3), 100-103. https://doi.org/10.1177/026455058403100308

Easton, H., y Matthews, R. (2011). Evaluation of the Inspire Women's Project, London South Bank

University. http://www.rogermatthews.net/images/research/inspire_women_s_project_evaluatio n_report.pdf

Fulham, L. (2019). International Trends in Community Alternatives to Incarceration: A Literature Review. Task Force on Women and Community Corrections, International Corrections and Prison Association (ICPA). https://icpa.org/wpcontent/uploads/2020/01/ICPA-Literature-Review-Final-Manuscript-1.pdf

Galbraith, S. (2004). So Tell Me, Why Do Women Need Something Different?. Journal of Religion \& Spirituality in Social Work, 23(1-2), 197-212.

Gelsthorpe, L. (2013). Working with Women in Probation: Will You, Won't You, Will You, Won't You, Won't You Join the Dance?. En P. Ugwudike y P. Raynor. (Eds.), What Works in offender compliance. International Perspectives and Evidence-Based Practice (pp. 279-294). Palgrave Macmillan.

Gelsthorpe, L. (2020). What works with women offenders? An English and Welsh perspective. En P. Ugwudike, H. Graham, F. McNeill, P. Raynor, F. Taxman y C. Trotter (Eds.). The Routledge Companion to Rehabilitative Work in Criminal Justice, (pp. 622-632). Routledge.

Gelsthorpe, L., Sharpe, G., y Roberts, J. (2007). Provision for women offenders in the community. Fawcett Society, Closing the inequality gap women since 1866. https://www.fawcettsociety.org.uk/provision-for-women-offenders-in-thecommunity

Howard League for Penal Reform (1999). Do Women Paint Fences Too?: Women's Experience of Community Service. (pp. 1-24). Biblioteca del Instituto de Criminología de Cambridge.

Instituto de la Mujer (2009). Análisis de la perspectiva de género en algunas estadísticas españolas y propuestas de mejora. Observatorio 16, Centro de Estudios Económicos Tomillo.https://www.inmujeres.gob.es/eu/observatorios/observIgualdad/estudiosInf ormes/docs/016-analisis.pdf

Igareda, N. (2006). Mujeres, integración y prisión. Boletín Criminológico, Instituto Andaluz Interuniversitario de Criminología, (86), 1-14.

Joiner, M. (2011). What is the impact of gender-responsive treatment on women offenders?. College of Professional Studies Professional Projects (Paper 31) [Tesis doctoral, Marquette University]. e-publications Marquette. https://epublications.marquette.edu/cps_professional/31/

Larrauri, E. (1992). La mujer ante el Derecho Penal. Revista de Derecho Penal y Criminología, (2), 291-310.

Larrauri, E. (Coord.). (1994). Mujeres, Derecho penal y criminología. Siglo XXI España Editores.

Revista Española de Investigación Criminológica

Artículo 3, Número 19 (2021)

https://doi.org/10.46381/reic.v19i1.443

Www.criminologia.net

ISSN: 1696-9219 
Malloch, M., y McIvor, G. (2011). Women and community sentences. Criminology and Criminal Justice, 11(4), 325-344. https://doi.org/10.1177/1748895811408839

Malloch, M., y McIvor, G. (2012). Women, Punishment and Social Justice: Human Rights and Penal Practices. Routledge Frontiers of Criminal Justice.

Masson, I., y Osterman, L. (2007). Working with female offenders in restorative justice frameworks. Effective and ethical practice. Probation Journal, 64(4), 354-371. https://doi.org/10.1177/0264550517728784

McIvor, G. (1998). Jobs for the Boys?: Gender Differences in Referral to Community Service. The Howard Journal, 37(3), 280-290.

McIvor, G. (2007). Paying Back - Unpaid Work by Offenders. En G. McIvor y P. Raynor, (Eds.), Developments in Social Work with Offenders (pp. 257-271). Research Highlights in Social Work 48, Jessica Kingsley Publishers.

Medina, J. (2005). El tratamiento al maltratador en el contexto comunitario como respuesta penal: consideraciones político criminales, Cuadernos Penales José María Lidón, 2, 183-208.

Navarro, C. (2018). L'execució de la pena de presó. Peculiaritats de l'execució penal femenina. Càtedra UAB-CICAC: Observatori Social i Econòmic de la Justícia. https://observatorijusticia.uab.cat/wpcontent/uploads/2020/02/Informeexecuciopenalfemenina.pdf

Nugent, B., y Loucks, N. (2011). Female Offenders in the community: The context of female crime. En R.Sheehan., G. McIvor y C. Trotter (Eds.), Working with women offenders in the community (pp. 1-25). Willan Publishing.

Patel, S., y Stanley, S. (2008). The use of the Community Order and the Suspended Sentence Order for Women. (pp.1-44). Centre for Crime and Justice Studies.

Penal Reform International (2016). Community service and probation for women: Lessons and recommendations based on a study in Kenya. Penal Reform International. https://www.penalreform.org/resource/community-service-probation-womenlessons-recommendations-based-study/

Petrillo, M. (2019). Women's desistance from crime: a gender-responsive, trauma-informed analysis. [Tesis doctoral, University of Portsmouth]. Portsmouth Research Portal. https://researchportal.port.ac.uk/portal/en/theses/womens-desistance-fromcrime(8de9d758-717d-497d-9fdf-9277d74596ab).html

Phoenix, O. (2017, julio 17). Kenya: Eight things to remember when implementing a gendersensitive approach to probation. Penal Reform International. https://www.penalreform.org/blog/eight-things-to-remember-when-implementing-agender/

Phoenix, O. (2018). Introducing a gender-sensitive approach to pre-trial assessment and probation: Evaluation of an innovation in Kenya. Probation Journal, The Journal of Community and Criminal Justice, 65(2), 184-200. https://doi.org/10.1177/0264550518771167

Prison Reform Trust (2018). Broken Trust: The rising numbers of women recalled to prison. Publications Prison Reform Trust. http://www.prisonreformtrust.org.uk/Portals/0/Documents/Broken\%20Trust.pdf

Revista Española de Investigación Criminológica

Artículo 3, Número 19 (2021)

https://doi.org/10.46381/reic.v19i1.443

Www.criminologia.net

ISSN: 1696-9219 
Reglas de Bangkok (2011). Reglas de las Naciones Unidas para el tratamiento de las reclusas y medidas no privativas de libertad para mujeres delincuentes. Organización de las Naciones Unidas.

Roberts, J. y Watson, G. (2017). Reducing the female admission to custody: exploring the options at sentencing. Criminology and Criminal Justice, 17(5), 546-567. https://doi.org/10.1177/1748895816684177

Ruiz, J.I. (2012). Metodología de la investigación cualitativa (5 $5^{\mathrm{a}}$ edición). Universidad de Deusto, Serie Ciencias Sociales, 15.

Sapouna, M., Bisset, C., Conlong, A-M., y Matthews, B. (2015). What Works to Reduce Reoffending: A Summary of the Evidence, Justice Analytical Services. Scottish Government, Crime and Justice, Social Research. https://www.gov.scot/publications/works-reduce-reoffending-summary-evidence/

Sheehan, R., McIvor, G., y Trotter, C. (2007). What Works with Women Offenders?. Willan Publishing.

Sheehan, R., McIvor, G., y Trotter, C. (2011). Working with Women Offenders in Community. Willan Publishing.

Smart, C. (1977). Women, Crime and Criminology: A Feminist Critique. Routledge \& Kegan Paul.

Vasilescu, C. (2019) La ejecución penal desde una perspectiva de género: una revisión bibliográfica con especial referencia a las medidas penales alternativas. InDret, Revista Análisis del Derecho, (2).

Vasilescu, C. (2020). La ejecución de medidas penales alternativas con perspectiva de género: análisis y recopilación de buenas prácticas con mujeres penadas. Centre d'Estudis Jurídics i $\quad$ Formació http://cejfe.gencat.cat/es/recerca/cataleg/crono/2020/execucio-mesurespenals/index.html

Wright, L., y Kemshall, H. (1994). Feminist Probation Practice: Making supervision meaningful. Probation Journal, 41(2), 73-80.

Worrall, A. (2003). "What works" and community sentences for women offenders. The Centre for Crime and Justice Studies, (53), 40-41. https://www.crimeandjustice.org.uk/publications/cjm/article/what-works-andcommunity-sentences-women-offenders

Worrall, A., y Gelsthorpe, L. (2009). "What works" with women offenders: the past 30 years. Probation Journal, 56(4), 329-345. https://doi.org/10.1177/0264550509346538

Yagüe, C. (2007). Mujeres en prisión. Intervención basada en sus características, necesidades y demandas. Revista Española de Investigación Criminológica, 5, 1-24. https://doi.org/10.46381/reic.v5i0.29 


\section{Agradecimientos}

Queremos agradecer en primer lugar a Ester Blay por los comentarios que hizo sobre una versión anterior a este trabajo. En segundo lugar, a la Direcció General d'Execució Penal a la Comunitat i Justícia Juvenil por autorizar la realización de esta investigación en los centros de medidas penales alternativas de Cataluña. En tercer lugar, agradecer a las/los revisoras/es anónimas/os por sus observaciones y comentarios, que, sin duda, han mejorado este artículo.

\section{Financiamiento}

Este estudio se inscribe en el proyecto "La discrecionalidad en la elección y la ejecución del castigo" (PGC2018-099155-B-I00), financiado por el Ministerio de Ciencia, Innovación y Universidades y el grupo de investigación reconocido por la Agencia de Gestión de Ayudas Universitarias y de Investigación de la Generalitat catalana Justicia Penal y Democracia (2017 SGR 1607).

Cristina Vasilescu es doctora en Derecho/Criminología por la Universidad de Girona, donde realizó su tesis doctoral sobre la ejecución penal de las medidas penales alternativas desde una perspectiva de género. Sus líneas de investigación incluyen, entre otras, la ejecución penal femenina, las medidas alternativas a la prisión y el análisis de género en el sistema de justicia penal.

https://orcid.org/0000-0003-1699-5623

José María López-Riba es doctor en Derecho/Criminología por la Universidad Pompeu Fabra, donde realizó su tesis doctoral sobre las identificaciones policiales. Actualmente es investigador postdoctoral en el Departamento de Derecho público de la Universidad de Girona. Además, imparte clases de diferentes materias en el Grado en criminología de la misma universidad. Sus líneas de investigación incluyen, entre otras, las actuaciones policiales, el derecho a la educación reglada en los centros penitenciarios, la discrecionalidad judicial y la selectividad del sistema penal.

https://orcid.org/0000-0003-0520-710X 